\title{
Au sujet d'un prétendu délit de blasphème en droit local alsacien-mosellan
}

\section{Jean-Marie Woehrling}

\section{OpenEdition}

\section{Journals}

Édition électronique

URL : http://journals.openedition.org/rdr/1076

DOI : $10.4000 /$ rdr. 1076

ISSN : 2534-7462

\section{Éditeur}

Presses universitaires de Strasbourg

Édition imprimée

Date de publication : 10 mai 2016

Pagination : 155-159

ISBN : 978-2-86820-954-2

ISSN : 2493-8637

Référence électronique

Jean-Marie Woehrling, «Au sujet d'un prétendu délit de blasphème en droit local alsacien-mosellan », Revue du droit des religions [En ligne], 1 | 2016, mis en ligne le 12 février 2020, consulté le 19 novembre 2020. URL : http://journals.openedition.org/rdr/1076 ; DOI : https://doi.org/10.4000/rdr.1076

\section{(c) (7) \&}

La revue du droit des religions est mise à disposition selon les termes de la Creative Commons Attribution - Pas d'Utilisation Commerciale 4.0 International - CC BY-NC 4.0. 


\section{AU SUJET D'UN PRÉTENDU DÉLIT DE BLASPHÈME EN DROIT LOCAL ALSACIEN-MOSELLAN}

\section{Jean-Marie WOEHRLING}

Président de l'Institut du droit local alsacien-mosellan

C'est devenu un lieu commun que de prétendre qu'il existe en droit alsacien-mosellan un « délit de blasphème » et de dénoncer à ce sujet l'archaïsme du droit local. La réalité est plus complexe.

Le texte "incriminé » est l'article 166 du Code pénal local usuellement traduit selon les termes suivants:

«Celui qui aura causé un scandale en blasphémant publiquement contre Dieu par des propos outrageants, ou aura publiquement outragé un des cultes chrétiens ou une communauté religieuse établie sur le territoire de la Confédération et reconnus comme corporation, ou les institutions ou cérémonies de ces cultes ou qui, dans une église ou un autre lieu consacré à des assemblées religieuses, aura commis des actes injurieux et scandaleux, sera puni d'un emprisonnement de trois ans au plus».

Ce texte est issu du Code pénal du Reich allemand adopté en 1871, lui-même inspiré en la matière du Code pénal prussien de 1854. Une lecture non informée de ce texte conclut que le «blasphème contre Dieu » est pénalement sanctionné. Mais les textes de droit, surtout s'ils sont anciens, doivent être lus avec une grille d'analyse juridique qui les réintègre dans leur contexte, dans la hiérarchie des normes et dans l'interprétation qui leur est donnée par la doctrine et la jurisprudence. 
Depuis la dernière partie du XIX ${ }^{\mathrm{e}}$ siècle, la doctrine interprète ce texte comme sanctionnant non le blasphème contre Dieu, mais l'agression contre les convictions religieuses d'autrui ${ }^{1}$. Le texte n'a semble-t-il jamais été appliqué par les tribunaux en Alsace-Lorraine (même s'il y a eu en ce sens des demandes qui ont été rejetées). Il a été utilisé plus fréquemment en Allemagne où il est resté en vigueur dans les mêmes termes jusqu'en 1969. Les tribunaux allemands lui ont donné le sens sus-rappelé dégagé par la doctrine : le délit sanctionné par ce texte réside dans une attaque contre les convictions religieuses d'autrui qui entraîne un trouble de la paix publique. Le texte modifié repris dans le Code pénal allemand actuel a également ce sens ${ }^{2}$. C'est aussi la signification donnée par la Cour européenne des droits de l'homme à des textes voisins applicables dans d'autres pays européens. Selon cette Cour, la liberté de religion, telle que protégée par l'article 9 de la Convention européenne des droits de l'homme, inclut une protection des convictions religieuses contre des attaques excessives ${ }^{3}$.

Ce texte législatif local doit désormais s'interpréter au regard des principes juridiques qui lui sont supérieurs. Les engagements internationaux de la France, ainsi que ses principes constitutionnels, garantissent la liberté d'opinion et d'expression, ce qui inclut le droit de porter un jugement critique à l'égard de toute conviction ou expression religieuse. L'article $1^{\text {er }} \mathrm{de}$ la Constitution française dispose que la République est laïque, ce qui signifie notamment qu'elle ne professe et ne peut faire prévaloir aucune conviction religieuse. Mais d'un autre côté, la Constitution affirme que la République « respecte toutes les croyances ». Pour qu'un tel respect soit effectif, il faut que le droit sanctionne les atteintes excessives aux croyances religieuses. De cet ensemble d'éléments, il ressort que l'article 166 ne peut plus se comprendre, malgré son libellé, comme comportant une sanction pénale du blasphème contre Dieu. Il assure une protection pénale de la liberté religieuse, laquelle implique une protection contre des agressions excessives contre les croyances d'autrui.

Mais que signifie une atteinte excessive à l'encontre d'une croyance religieuse ? On ne saurait retenir un critère subjectif tenant à l'intention de l'auteur de la critique ou au ressenti de celui qui se considère blessé dans sa conviction religieuse. Le critère doit être objectif et fondé sur des

1. Liszt F. von., Lehrbuch des deutschen Strafrechts, Berlin, Walter de Gruyter, 1922, p. 411.

2. Woenrling J.-M., « Le "délit de blasphème" en droit local : un archaïsme ? », Revue du droit local, $n^{\circ} 67,2013$.

3. CEDH, 20 sept. 1994, n 13470/87, Otto Preminger-Institut c/ Autriche. 
considérations d'ordre public. C'est pourquoi la doctrine et la jurisprudence allemandes ont pris comme critère du caractère excessif de l'agression contre une conviction religieuse le trouble occasionné à la paix publique. En droit français, le terme consacré est celui de trouble à l'ordre public.

À cet égard, il faut rappeler que la loi sur la presse du 29 juillet 1881 (art. 24), qui est applicable en Alsace-Moselle, prévoit une peine de 5 ans d'emprisonnement et de 45000 euros d'amende à ceux qui auront provoqué à la discrimination, à la haine ou à la violence à l'égard d'une personne ou d'un groupe en raison de leur religion. Selon l'article 29 alinéa 2 de la même loi, toute expression outrageante est une insulte punissable pénalement. L'article 32 de cette loi prévoit un an de prison et 45000 euros d'amende pour la diffamation commise envers une personne ou un groupe à raison de leur appartenance à une religion. Enfin, l'article 33 punit de 6 mois d'emprisonnement et de 22000 euros d'amende les injures proférées envers une personne ou un groupe à raison de l'appartenance ou de la non-appartenance à une religion. Ces dispositions remontent à une loi du $1^{\text {er }}$ juillet $1972^{4}$.

Ces dispositions de la loi française sur la presse ont donné lieu à diverses procédures, dont on peut citer quelques exemples :

- le fait de publier une caricature représentant une religieuse d'aspect grotesque occupée devant un sapin de Noël à gonfler avec une pompe à vélo un Christ en croix, même s'il est de nature à heurter la sensibilité des catholiques ne constitue pas une invitation à la haine ou une provocation ${ }^{5}$;

- par contre, une série de caricatures de la revue "La Grosse Berthe » représentant Jean-Paul II avec un travesti brésilien, un prêtre noyant un enfant dans un baptistère et une femme nue éventrée par un crucifix a été considérée comme une injure relevant de l'article 24 de la loi de $1881^{6}$;

- de même ont été considérés comme une diffamation religieuse des propos violents tenus à l'encontre du Carmel d'Auschwitz ${ }^{7}$;

4. Comme le relève Brigitte BASDEVANT-GAUdEMET (« Histoire juridique du blasphème : péché, délit, liberté d'expression? », RDP 2015, p. 309), dans sa rédaction initiale, la loi de 1881 ne contenait aucune référence à la religion. C'est un décret-loi du 21 avril 1939 qui modifie l'article 32 en vue d'introduire la répression de la diffamation commise envers un groupe de personnes qui appartiennent à une religion déterminée. La loi ${ }^{\circ} 72-546$ du $1^{\text {er }}$ juillet 1972 relative à la lutte contre le racisme introduit le régime actuellement en vigueur, l'excitation à la haine n'étant plus retenue comme condition pour la mise en œuvre de la répression (Foulon-Piganiol J., D. 1970, chron. p. 163 et D. 1972, chron. p. 26).

5. Cass. crim., 7 déc. 1993, AGRIF, Dr. pén. 1994, comm. n 80, note VÉron.

6. Cass. $2^{\mathrm{e}}$ civ., 28 févr. 1996, AGRIF, nº 93-20.663.

7. Cass. crim., 14 juin 2000, $n^{\circ}$ 99-80.043. 
- dans l'affaire de la représentation de la Sainte Cène aux fins de publicité pour une marque de vêtements, la Cour de cassation, contrairement aux premiers juges et aux juges d'appel, a considéré qu'il n'y avait pas insulte à l'égard de la religion chrétienne ${ }^{8}$. On pourrait considérer que les dispositions susmentionnées de la loi sur la presse sont très proches de l'article 166 dans l'interprétation proposée ci-dessus. Il existe cependant un débat dans la doctrine juridique française sur le point de savoir si le respect des convictions religieuses est suffisamment garanti par le droit français ${ }^{9}$.

Certes, on peut se demander si la sanction pénale est bien adaptée à la protection des convictions religieuses. Le fait que le texte de l'article 166 du Code pénal local n'ait apparemment jamais été mis en œuvre en AlsaceMoselle depuis 1918 semble attester de la faible utilité de cette disposition. La condamnation pénale de l'agression contre des convictions religieuses a un intérêt pratique limité, car elle intervient après les événements préjudiciables. Son principal apport est de stigmatiser symboliquement les comportements dangereux pour la paix publique et de punir les actes marqués par la mauvaise foi, une intention de nuire, ou du moins une imprudence coupable.

L'action civile qui permet de recourir à des procédures d'urgence (action en référé tendant à empêcher ou à arrêter les comportements hostiles) paraît, d'un point de vue pratique, plus adaptée que l'action pénale pour éviter l'atteinte aux croyances. Mais la Cour de cassation a refusé d'admettre que les abus de la liberté d'expression prévus et réprimés par la loi du 29 juillet 1881 puissent être réparés sur le fondement de l'article 1382 du Code civil ${ }^{10}$. C'est donc l'indigence du droit civil qui conduit à l'utilisation peu satisfaisante du droit pénal.

D'une façon plus générale, ce sujet est toujours difficile : entre le sentiment de blessure ressentie par les personnes animées d'une forte conviction religieuse et l'affirmation extensive de la liberté d'expression, il ne revient au juge d'intervenir qu'en cas de trouble grave à la paix publique, c'est-à-dire quand l'offense contre la religion risque de conduire à la violence publique.

Il convient encore de relever que l'article 166 comporte aussi une protection contre les actes scandaleux commis dans les lieux consacrés aux assemblées religieuses. Cette protection existe également en droit général : la loi de 1905, prévoit que seront punis de peine d'amende les auteurs de menaces qui auront

8. Cass. $1^{\text {re }}$ civ., 14 nov. 2006, n 05-15.822 05-16.001.

9. Dreger E., « La cour de Cassation et le désarroi des croyants », D. 2007, p. 2072.

10. Cass. $2^{\mathrm{e}}$ civ., 8 mars 2001, n $98-17574$, JCP G 2002, I, p. 546. 
déterminé un individu à s'abstenir d'exercer un culte ou à faire partie d'une association cultuelle ou les actes d'empêchement de l'exercice d'un culte. Il est probable que c'est pour se substituer à ces dispositions, non introduites en Alsace-Moselle, que le législateur de 1924 a conservé les dispositions de même nature, mais plus larges, du Code pénal local.

Que faut-il faire de l'article 166 ? Certains pensent qu'il n'est plus applicable au motif qu'il n'a pas fait l'objet d'une traduction en français publiée de manière officielle (cela paraît être la position du ministère de l'Intérieur qui se fonde sur une décision du Conseil constitutionnel, qui a soulevé cette question de la traduction des textes locaux d'origine allemande ${ }^{11}$ ). Sa suppression formelle ne causerait pas de difficultés graves puisque la disposition n'est pas appliquée. Mais elle ne règlerait pas le problème de la définition des limites de la liberté d'expression et laisserait ouverte la question d'une protection appropriée des convictions religieuses. Elle créerait de surcroît un vide en ce qui concerne la sanction des actes scandaleux dans les lieux consacrés aux assemblées religieuses. Une modification de l'article 166, comme cela a été réalisé en Allemagne fédérale, serait raisonnable en théorie, mais dans le contexte actuel, peut-on escompter un débat serein et équilibré sur un tel sujet au Parlement? Ne vaudrait-il pas mieux, en fin de compte, s'occuper des vrais problèmes que de discuter d'une disposition locale qui n'a jamais été appliquée ? Sachant qu'une appréciation au cas par cas est indispensable, le plus sage ne serait-il pas de laisser les tribunaux faire usage avec intelligence des textes existants ?

Au terme de cette analyse, on peut constater que le « délit de blasphème » n'existe pas en droit local et que la protection qu'il organise contre les attaques abusives des convictions religieuses ne présente qu'une originalité limitée par rapport aux dispositions du droit général. Une réécriture moderne de ce texte pour qu'il soit mieux compréhensible serait certes souhaitable mais paraît irréalisable.

11. Cons. const., déc. 30 nov. 2012, n² 2012-285 QPC, Christian S. 